\title{
Simulations of Core Convection Dynamos in Rotating A-type Stars
}

\author{
Matthew Browning, Allan Sacha Brun, and Juri Toomre \\ JILA, University of Colorado, Boulder, CO, USA 80309-0440
}

\begin{abstract}
We have conducted preliminary numerical simulations of a core convection dynamo operating within an A-type star of two solar masses. Convection within the core clearly can admit magnetic dynamo action. Magnetic field strengths in our three-dimensional simulations grow by many orders of magnitude, from an initial seed field to kilo-Gauss levels. We discuss the differential rotation and magnetic field sustained in our simulations.
\end{abstract}

\section{Motivation}

A-type stars possess cores of vigorous convection. Such convection, coupled with high electrical conductivity and rotation, is likely to give rise to dynamo action deep within these stars. Strong surface magnetic features have been observed in a subset of the A-type stars. A favored origin for these surface fields is primordial, but if fields generated within the core are susceptible to magnetic buoyancy instabilities, they might also contribute to the observed surface phenomena. Additionally, magnetic fields within the core might modify the differential rotation achieved in the star, as magnetic field lines anchored in the rotating core drag material in the radiative zone above.

\section{Approach}

We utilize our 3-D anelastic spherical harmonic code (ASH), operating within spherical geometries, on massively parallel supercomputers. Our computational

Figure 1. Snapshot of the azimuthal component of magnetic field in viewing the convective core. The axis of rotation is oriented vertically in this depiction. Positive values (magnetic field directed to the right) are rendered as light tones; negative values are dark. The flow is complex and highly time-dependent.

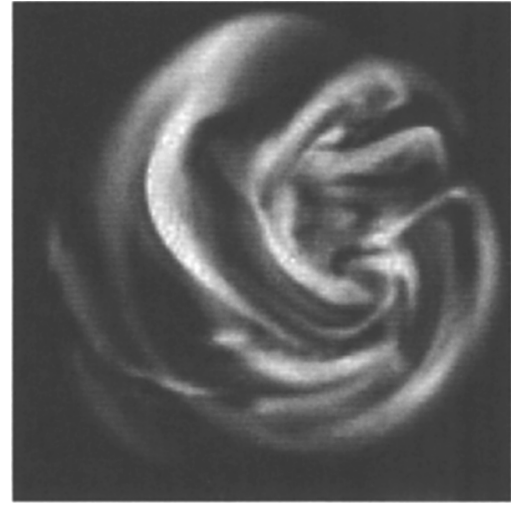


Figure 2. Angular velocity, averaged in time and longitude. A strong differential rotation is maintained, much as in the progenitor non-magnetic case. The magnetic field lessens the differential rotation somewhat, but a central column of strikingly slow rotation persists.
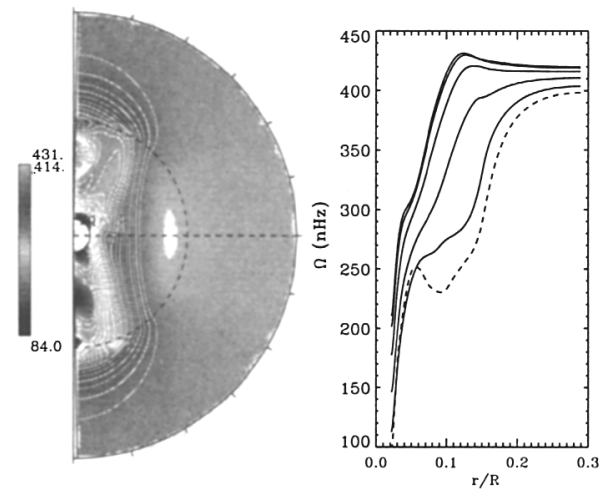

Figure 3. Growth of magnetic energy density (ME) with time, starting from a mature nonmagnetic case. Shown also are the other kinetic energy densities: differential rotation (DRKE), meridional circulation (MCKE), and total kinetic energy (KE), all relative to the rotating frame (at $414 \mathrm{nHz}$ ). The magnetic energy density grows to a value greater than MCKE and DRKE, and to about $30 \%$ of KE.

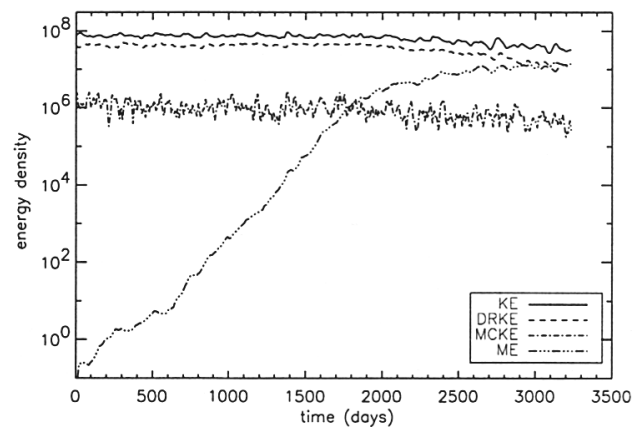

domain extends over the inner $30 \%$ of the star in radius, including the convective core (occupying the inner $15 \%$ of the star) and a portion of the overlying radiative zone. The innermost $2 \%$ of the star is excluded from our computational domain for numerical reasons.

\section{Results and Conclusions}

- The preliminary simulations here indicate that vigorous core convection within a prototypical A-type star, rotating at the relatively slow solar rotation rate, can result in sustained magnetic dynamo action.

- The strong differential rotation realized in our progenitor non-magnetic case (Brun, Browning, \& Toomre, this volume) persists, but is lessened.

- Our simulations cannot yet establish whether strong magnetic fields generated within the core might become unstable to magnetic buoyancy instabilities and rise to the surface.

- We plan to explore different regimes for the relevant simulation parameters (such as rotation rate, magnetic diffusivity, and viscosity) to determine the sensitivity of the flows and magnetic fields established here to those parameters. 